\title{
Eye Tracking in Maritime Immersive Safe Oceans Technology
}

\author{
Mika Luimula $^{1}$, Evangelos Markopoulos ${ }^{2,1}$, Johanna Kaakinen ${ }^{3}$, \\ Panagiotis Markopoulos ${ }^{4}$, Niko Laivuori ${ }^{1}$, Werner Ravyse ${ }^{1}$ \\ ${ }^{1}$ Turku University of Applied Sciences, Turku Finland \\ ${ }^{2}$ University College London, London, UK. \\ ${ }^{3}$ University of Turku, Turku Finland \\ ${ }^{4}$ University of the Arts London, London UK \\ mika.luimula@turkuamk.fi; e.markopoulos@ucl.ac.uk; johanna.kaakinen@utu.fi; p.markopoulos1@arts.ac.uk; \\ niko.laivuori@edu.turkuamk.fi; werner.ravyse@turkuamk.fi
}

\begin{abstract}
This paper presents the integration of eye tracking in the MarSEVR (Maritime Safety Education with VR) technology to increase the precision of the trainee focus on delivering the learning episodes of the technology with enhanced impressiveness and user engagement. MarSEVR is part of the Safe Oceans concept, a green ocean technology that integrates several VR safety training applications to reduce maritime accidents that result into human casualties, sea pollution and other environmental damages. The paper indicates the research delivery architecture driven by Hevner's design science in information systems Research for usability, use experience (UX) and effectiveness. Furthermore, this technology integration is approached from a game design perspective for user engagement but also from a cognitive and neuroscience perspective for pedagogical use and purposes. The paper addressees the impact of the eye tracking technology in the maritime sector operations, training market, and competitive research. Lastly areas of further research are presented and the efforts to link and align finger tracking and hand recognitions technologies with eye tracking for a more complete VR training environment.
\end{abstract}

Keywords: Eye tracking, Virtual training, Virtual Reality, Cognitive Science, Neuroscience, Maritime safety training, Shipping.

\section{INTRODUCTION}

Maritime is a sector which intensively applies ICT driven educations. Simulator centers with several command bridge environments offer natural learning environments for maritime students and the seafarers. Due to the nature of maritime there is a need for onboard training and in our previous papers we have introduced various training episodes to be used with VR headsets. Based on our first experiments [1] we have identified usability challenges and needs for improvements in user experience.

Prior presenting the need of the maritime sector to adopt effective and continuous training, especially on safety issues, it is important to understand the size of the shipping industry and the role of virtual reality in maritime safety education. The international shipping industry transports nearly $90 \%$ of the world trade with world fleet to be more than 60,000 merchant ships, registered in over 150 nations, manned by nearly 2 million seafarers, transporting every kind of cargo. Despite the improvements on ship design, development, port management and on the overall integration of advanced technology in the vessels, shipping still suffers from significant losses with the cargo vessels to account nearly $35 \%$ in 2018 primarily in the Asian region (China, Korea, South East Asia and Japan). Furthermore, foundered (sunk/submerged) is the cause for $53 \%$ of the total losses the last 10 years, and $68 \%$ in 2018 [2]. In the maritime industry the safety of the vessels is critical and relies heavily on continuous safety education. On the other hand maintaining such training on-shore restricts the effectiveness of the industry due to the training costs, the time needed for the seafarers to spend out of the vessel, the rarity of state of the art simulations and the waiting time to access them [3].

Virtual reality maritime safety training can contribute on resolving such challenges. Industry experts believe that immersing the learner in VR environments provides deeper understanding of the processes and procedures required to undertake in a given situation. The $360^{\circ}$ visualization features supported with haptic motion controllers, simulate real-world tasks that must be performed by the competent seafarers [4]. This has shifted maritime education to alternative methods with the shipowners to be mode adaptive to e-learning, and the ship providers to develop VR-based training to help shipping managers overcome operational challenges.

In this paper, we will report our first experiences utilizing eye tracking sensors in MarSEVR application. Eye gaze reflects the allocation of attentional resources and can be used as an index of the mental processes underlying behavior in VR environments [5]. The decisions of where and when to look at are guided by the task that is being completed, and research suggests task-irrelevant objects or locations are only rarely fixated [5,6]. Our multidisciplinary team consisting VR developers, graphical designers, and maritime specialists has now been extended with cognitive neuroscientists. This is not the first study in which we have combined virtual reality and neuroscience. In NeuroCar project (Figure 1), we developed a solution utilizing virtual reality in driving inspection. We were able to prove that VR headsets can be used in laboratory conditions to detect the right-side perceptual bias during a virtual driving task [7]. 


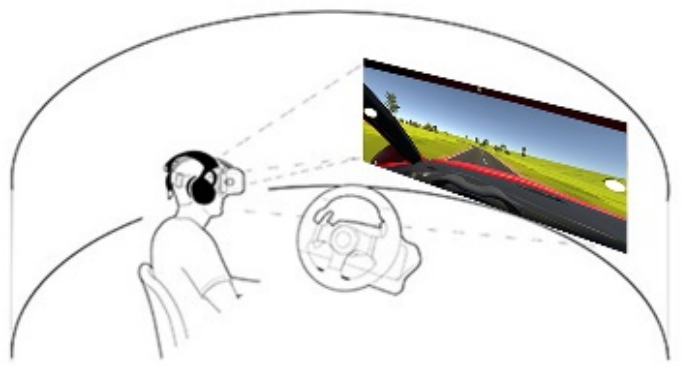

Figure 1. Virtual reality in driving inspection [8].

In Markopoulos \& Luimula [9], we have introduced the Immersive Safe Oceans Technology with various virtual training episodes developed for maritime safety training. This technology will operate in the future as a training jukebox with each scenario to be executed upon schedule or demand (Figure 2). As of today we have developed training episodes for command bridge, ship engine, crane, and fire safety concepts so far. These episodes are examples of various use cases for onboard training. This technology can be brought onboard in any type of vessel and all training data can be collected and analyzed. Eye tracking will be an essential new feature in data analysis and in this paper we will show how eye tracking can be used to track and trace human behavior in stressed conditions and to improve usability and user experience in training episodes.

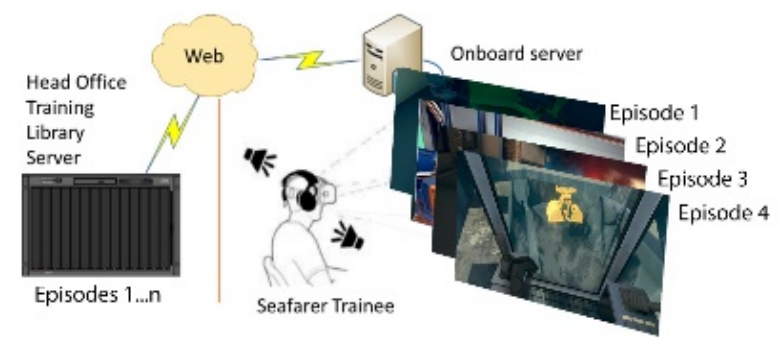

Figure 2. Training libraries in maritime safety [9]

\section{EYE MOVEMENT BEHAVIOR IN VIRTUAL REALITY}

According to Hayoe and Ballard [5], eye movements serve functions at three levels of abstraction. At the behavioral level, eye movements provide visual information needed in performing the task at hand. For example, when reaching out to open a door handle, eye movements are probably guided to the proximity of the handle to support the accurate hand movement towards it. At the level of resource allocation, eye movements indicate the selection of the subtask to be performed. For example, if the goal is to walk into a room, it may involve multiple subtasks that have to performed in order to successfully enter the room. One needs to approach the door, grasp its handle, open the door, and navigate through the opening without hitting one's head on the door frame or tripping on the doorstep. Eye gaze reflects which one of the subtasks is attended at a given moment, as the limitations of attentional capacity prevent people of focusing on more than one (or, very few) things at a time.
Finally, at the contextual level eye movements reflect the active set of actions that are appropriate considering the environment and the task to be completed. For example, if we notice that the door handle is stuck and does not turn down, we need to change our initial action plan and search for an appropriate solution, such as checking if there is a lock on the door and if it can be opened. These three levels of abstraction open new possibilities to improve the pedagogy used in our existing virtual training episodes such as Fire Safety Onboard (Figure 3) introduced in [9].

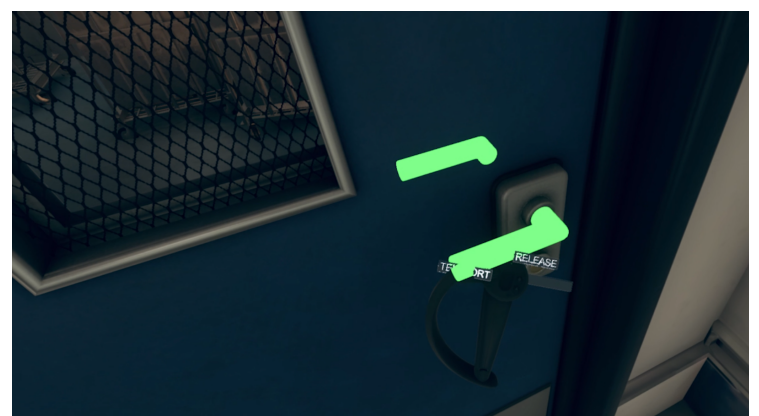

Figure 3. Three levels of abstraction to be used in Fire Safety Onboard episode.

As eye gaze is heavily influenced by the task and its demands, the analysis of eye movement patterns requires understanding of the task structure. Higher order goals (e.g. entering a room) can be divided into smaller subtasks, which may be either parallel or sequential, depending on the nature of the task to be performed $[5,6]$. If the subtasks are parallel, eye gaze reflects the shifts of attention between the tasks (i.e., the preferred task at a given time) and actions needed to complete them. If the subtasks are sequential, then eye gaze reflects the order in which the actions are performed. For example, if somebody looks at the door handle when performing a task of entering a room, they probably are planning to grasp the handle and use the information obtained with eye gaze to guide hand movement. Eye movements are pro-active - they usually precede the actions to be completed and are predictive in nature $[5,6,10]$.

The eye movement patterns that efficiently support task completion are learned with practice $[2,3]$, and eyes are directed to objects or locations that have proven optimal for completing a task. Moreover, eye movement control is influenced by the mental representation of the task and its structure. Eye movement patterns should thus change as a result of learning: one can learn what is the best time and location to gaze at during task completion, as well as the steps required to successfully perform a task. This means that an analysis of eye movement patterns can be used to track learning during e.g. VR training programs.

TABLE II. GAZE DATA LINKED WITH GAME OBJECTS

\begin{tabular}{|l|c|c|}
\hline \multicolumn{1}{|c|}{ ObjectName } & FramesCount & Percentage \\
\hline wall_1 & 401 & 9,130237 \\
\hline door & 103 & 2,345173 \\
\hline door_handle & 245 & 5,578324 \\
\hline
\end{tabular}


TABLE II. GAZE DATA COLLECTION IN OUR VIRTUAL REALITY APPLICATION

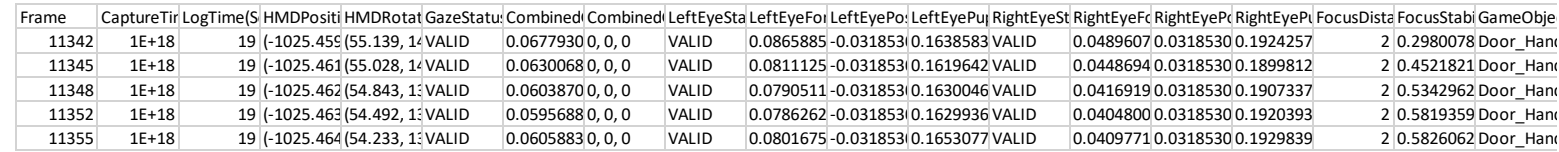

To be able to analyze eye movement patterns in our virtual training episodes in the future we have linked gaze data with game objects (Table 1). In this paper, we have used Varjo's VR-2 Head Mounted Display. The technical description of our new MarSEVR application is introduced in the following chapters. To follow required steps to complete given tasks in our training episodes we can analyze head movements based on values (HMDPosition, and HMDRotation) together with eye position, eye direction, pupil size, and focus stability presented in Table 2 .

When analyzing eye movement behavior, different measures from the raw gaze coordinate data are extracted. Which measures are computed naturally depends on the research question, task that was performed and the characteristics of the VR environment? Typical measures of eye movement behavior in naturalistic learning environments, including VR environments, involve defining first areas of interest (AOI's) in the visual environment (see e.g. [11]). For example, based on a task analysis, task-relevant and irrelevant targets can be identified and defined as AOI's. Next, one would compute measures like dwell time directed on a specific AOI during task performance, transitions between AOI's, and the timing between the eye gaze directed to different AOI's and the action to be completed. These analyses could be timelocked to specific target events or actions. After these measures are computed, they can be used to numerically describe eye movement behavior in the VR environment for different participants, and subjected to statistical analyses to infer for example differences in eye movement patterns of the users pre- and post-training. Data visualizations, like dynamic heatmaps based on gaze data, help in interpreting the results.

From the perspective of psychology, VR environments provide an interesting platform for doing research on the cognitive processes underpinning human learning and behavior [12]. However, the research of the basic cognitive processes in the VR is just emerging. The results of an early study by Kit et al. [13] indicated that when participants were engaged in a continuous visual search task within a VR environment for three days, they quickly memorized the location of the objects in the environment, which benefited the search task. The analysis of the eye gaze patterns showed that incidental fixations made on objects did not help in subsequent search, suggesting that without a specific task the fixated objects are not permanently coded to memory. On the other hand, Helbing et al. [14] found that in VR environments, incidental viewing might result in better learning outcomes for the features present in the environment (memory for object identity and location) than directed memorization ([14]. Understanding the relationship between viewing behavior, attention, and memory processes in VR is crucial to develop environments that optimally support learning. There thus is a pressing need for experimental research on these topics.

\section{THE MARSEVR COMMAND BRIDGE APPLICATION}

MarSEVR (Marine Safety Education with Virtual Reality) is a technology for virtual training on command bridge operations. The technology does not indicate any training limitations comparted to traditional command bridge simulators on its operations and learning content [15]. It adopts VR and game design principles to implement, immersive training scenarios in an engaging and immersive way that contributes to better understand the right decision-making factors in a gamified environment (Figure 4).

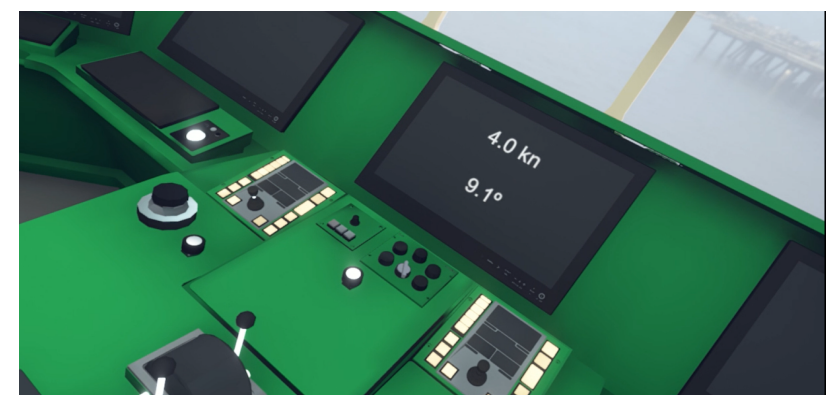
Figure 4. The MarSEVR command bridge realistic surrounding environment.

The technology is composed from training scenarios practicing situation awareness and decision making. The replication of the ship bridge environment allows the creation of different training scenarios in levels of difficulty by adjusting the natural phenomena (wave, rain, etc), or the number of obstacles at the sea (other vessels, fishing boats, etc) shown in Figure 5.

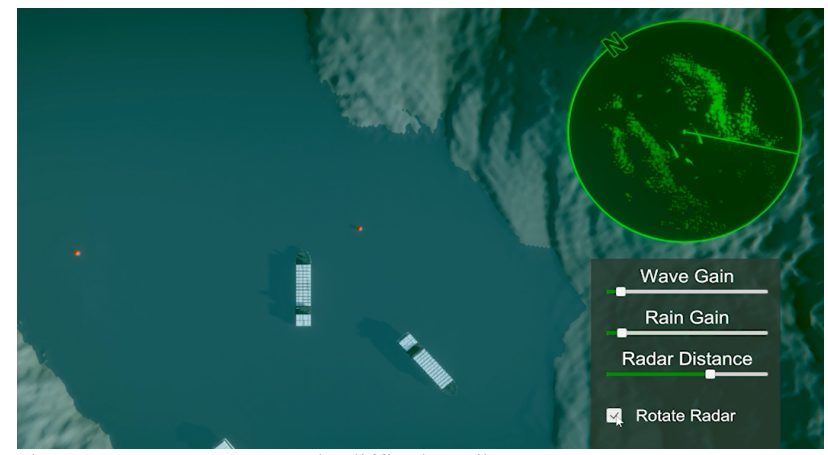

Figure 5. MarSEVR scenario difficulty adjustments 
The first training scenario developed for MarSERV is related with a watch change and collision avoidance situation. The learning goal for the training is to demonstrate the needed attention to the various equipment status and settings and to take actions need to avoid a possible collision. The technology has been tested with industry experts and command bridge offices at leading shipping companies and from credible shipping journalists.

MaSEVR has been characterized as disruptive innovation that transforms maritime safety training and education [16]. Furthermore, its innovative research received awards from academic international journals and conferences.

\section{RESEARCH DELIVERY ARCHITECTURE}

The aim of this paper is to introduce our roadmap for more immersive training episodes in maritime safety training and our current RDI activities especially related to eye tracking in virtual reality. The research delivery architecture activities related to Immersive Safe Oceans Technology is presented in Figure 4 are designed according to Hevner's information systems framework [17] adopted to frequent industrial cooperation and rigorous academic research activities. The utilization of the eye tracking in maritime safety training enables us to track and trace more accurately human behavior but in the same time opens new ways to improve usability and user experience in training episodes. Our research and development activities are designed in a close cooperation with industrial and academic partners such as Varjo Ltd, University of Turku (Turku EyeLabs). This study can be seen as one of the testgenerate cycles we run at the moment. Eye tracking is just one important additional tool in our research while focusing on usability, user experience, and effectiveness studies.

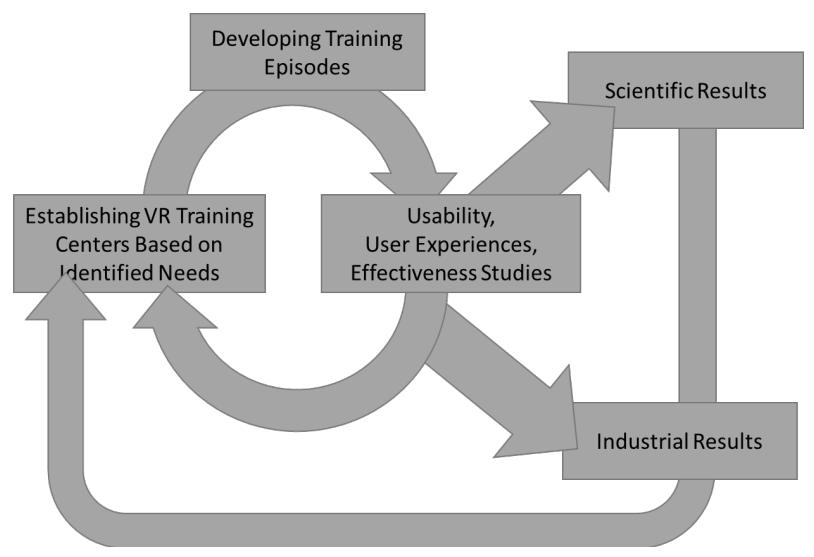

Figure 6. Eye tracking to be used for effectiveness studies (cf. [17])

Later in this paper, we will introduce a new version of MarSEVR application utilizing eye tracking to be able to focus more deeper on effectiveness studies. This application has been developed in test-generate cycles where feedback has been received both from industry and academy. In practice, industrial feedback has been collected based on user-centric design principles to identify requirements and needs and using agile software development methods (especially scrum) to enable frequent communication and steady progress in the development. In the same time, from scientific point of view research focus has so far been mainly on usability and user experience studies.

User experience (UX) is a particular design discipline that covers all the end-user's interaction from the company to its services, and products [18]. We further accept the experience to comprise the process and outcome of user engagement with a technology-driven environment. In our case the environment is formed by the VR headset that serves the learning goal within the maritime domain. If users have a poor experience with the VR headset, or they are dissatisfied with the learning outcomes they have set out to achieve, they will be reluctant not only to re-engage with the current environment but also with subsequent learning environments served up through VR. Hence, the importance of UX studies within our research design is highlighted.

Although usability certainly impacts UX, usability studies give more specific insight on the system interaction barriers (for example, complex controller layout or an unclear user interface) users must overcome, rather than the perceived experience. Without thoroughly studying the usability of VR learning scenarios, we would run the risk of detracting from contextual learning outcomes by cognitively overloading the user with learning and attempting to remember how to use the VR environment.

Both usability and UX studies are set in the rigors that produce scientific results while attending to our usercentric design principals by being an integral part of the iterative design-develop cycles. Usability and UX study results are essential in guiding the development of VR training episodes that must deliver unbiased effectiveness reports [19]. Poor usability and low UX will most certainly undermine the effectiveness of a learning episode. Effectiveness studies essentially capture how conducive the VR training episodes are in transferring knowledge to the user. This has industrial value where an effectiveness study with positive results provides a guarantee of knowledge transfer. This means that our industrial partners can be assured that their employees will return from a VR training episode with the necessary knowledge to perform adequately the tasks addressed in that specific episode. By ensuring that our VR training episodes have a positive user experience with a high usability rating, we can be certain that the results of the effectiveness studies are not skewed.

In the event of an effectiveness study showing a negative outcome whereby there is an insufficient level of knowledge transfer, we would revisit the iterative development phase with a new episode design that still concentrates on the needs and context analysis for that episode. Vice versa, when the scientific results from the usability, UX and effectiveness studies demonstrate satisfactory industrial results, we assess the context again. If all needs within the context have not been met, we restart the process of producing a next learning episode that complements the existing episodes within the current context. Otherwise, an entirely new suite of episodes for a new context will begin. 


\section{INTEGRATION OF THE EYE TRACKING SENSOR IN MARSEVR APPLICATION}

The integration of the eye tracking technology in MarSEVR is part of the MarISOT (Maritime Immersive Ocean Technology) strategy presented in Figure 7.

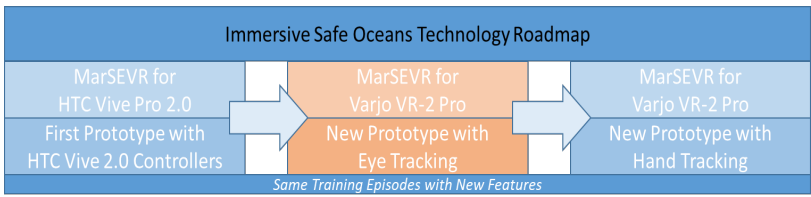

Figure 7. Technology roadmap to increase the immersiveness.

In our experiments we used the Varjo VR-2 Pro VR headsets with two cameras recording in the infrared spectrum and capturing images of the eyes at 100 frames per second with a relatively high resolution of $1280 \times 800$ pixels [20]. These cameras inside the headsets, one for each eye, capturing images of the eyes at 100 frames per second. The sensors used in headsets have a very high resolution $(1280 \times 800$ pixels $)$ compared to most eye tracking solutions. Varjo headsets do all of the recording in the infrared (IR) spectrum.

The Varjo VR-2 Pro VR headsets have now been integrated into the MarSEVR command bridge application using Unity game engine (version 2018.3.14f1), the Varjo plugin (version 2.3.0.3) for Unity alongside SteamVR plugin (version 2.2.0). The original MarSEVR application has been developed with Unity game engine already in 2019 and in previous studies we were using HTC Vive Pro VR 2.0 headsets. Varjo plugin handles the rendering and SteamVR plugin provides controller interaction. In this study, we were using HTC Vive Pro 2.0 controllers. In addition, Varjo Base software was needed to use the Varjo headsets on Microsoft Windows OS.

The Varjo plugin on the top of the SteamVR offers tools to render image to the Varjo headset, to detect user's gaze, to calibrate eye tracking manually, and to configurate the eye tracking. Calibration is needed every time while the user puts headset back on since even a slight change in headset's position will affect in the accuracy of the eye tracking. The Varjo VR-2 headset has internal eye tracking in the frequency of $100 \mathrm{~Hz}$ but in can be configurated. In this study, we logged eye data once every frame in Unity.

Command bridges are an interesting use case to utilize eye tracking due to its complex operation environment. We have seen that especially inexperienced users with no gaming experiences, have difficulties to act in virtual reality environments without extra guidance. The Varjo's headsets eye tracking sensors offers the ability to track and trace human behavior. In addition, users can have new ways to interact in the virtual environment. In this case we present how eye tracking data can be used to improve some usability issues and in the same time offer better user experience. Command bridge consist tens of interactive objects. With the Varjo's Unity plugin developers are able to send gaze ray commands to highlight interactive objects in users focus area. In Figure 8, user's gaze is focused on ship's wheel and it is highlighted with white color.

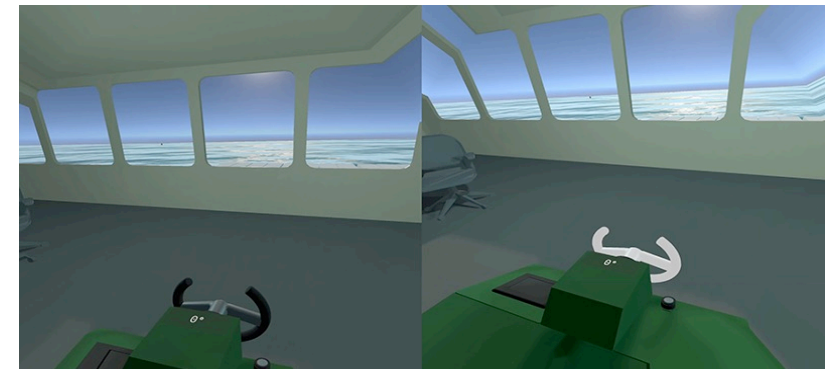

Figure 8. Ship's wheel highlighted when user focusing eye on it.

Based on our first experiences the Varjo's eye tracking sensors are relatively accurate and different interactive objects same time in the view can be recognized quite reliable (Figure 9). This offers for developers an opportunity to create training episodes where only some of the command bridge controllers will be active during this specific training session.

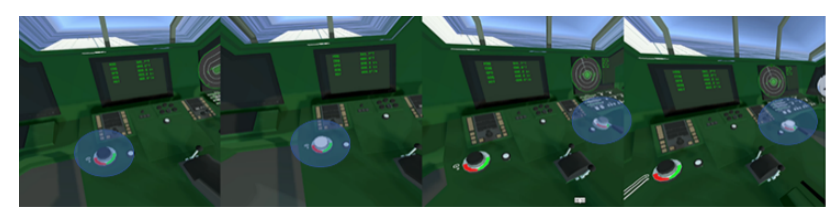

Figure 9. User turning eye from one focus point to another.

\section{IMPACT TO THE MARITIME SECTOR AND FUTURE DIRECTIONS}

The integration of the eye tracking technology in the MarSEVR was one of the requirements of the maritime industry experts who tested previous versions of the technology. It has been indicated that the confidence of the bridge commander and the rest of the crew is reflected through their eyes on the actions taken to perform their operations. Hesitation, thinking delays, second guessing, wondering, and other feelings can be expressed and identified by the eye movements. When on-board, sailing across the oceans it is the confidence above the skills that secure safe journeys. The eye tracking technology provides more than understanding the conference of the seafarers when performing a task. It extends towards understanding the mental state that impacts the psychology of the seafarers which is reflected on their eye movements. This is a major issue for the shipping insurance companies that play a critical role on the effective and profitable operations of the shipping industry. Virtual training that can extend beyond the safety technical skills of a seafarer is in demand by various parties involved in the industry.

In an analysis made by Allianz Global Corporate \& Specialty (AGCS) on more than 230,000 marine insurance industry claims between July 2013 and July 2018 indicate claim value of almost $\$ 10 \mathrm{bn}$. This analysis also shows that ship sinking/collision incidents are the $16 \%$ of the value of all claims, and the most expensive cause of loss for insurers as they are equivalent to more than $\$ 1.5 \mathrm{bn}$. Likewise the value of claims from machinery damage incidents was $\$ 1 \mathrm{bn}+[12]$.

With the eye tracking technology being integrated on MarSEVR who operates in the sphere of collision avoidance safety training, this insurance issue is being 
addressed. This brings MarSEVR a step closer to the expectations of the maritime and shipping sector's requirements for virtual training on safety issues.

Parallel research is being conducted by the MarSEVR research team to integrate in the technology Hand Recognition and Finger Tracking as well. The two projects run in parallel and once completed they will be integrated for the synchronization of the eye with the hand to achieve higher levels of immersiveness. The hand recognition and finger tracking has also been a requirement from the shipping industry experts who tested the previous version of the application. Operating hands free provides higher freedom of movement, increases concentration of the task to be performed and reduces mistakes that can be done from attention disturbance on handling the controls properly. This is highly related with the eye tracking technology presented in this work as the eye movement can be corelated with the hand movement and together to record precisely the command bridge officer's behavior.

This coordination will be examined as the next stage of this research and the results will be critically analyzed from the dimensions of user engagement, immersion, cognitive and neuroscience pedagogy, and training effectiveness. Furthermore this work intends to be extended on other VR Safety Training applications developed by this research group on engine room maintenance, fire safety and crane safety which compose, together with MarSEVR, the MarISOT (Maritime Immersive Safe Oceanites Technology) portfolio [6].

\section{CONCLUSIONS}

In this paper, we have shown how we can collect eye tracking data and analyze eye movement behavior during virtual maritime safety training. Further studies will require a multidisciplinary team consisting of cognitive neuroscientists, and maritime specialists / pedagogists working closely with our VR development team. We are already able to collect gaze data including eye position, eye direction, pupil size, and focus stability. In addition, we can combine game object data with gaze data collection.

This study can be used next in designing new learning scenarios where user will be given tasks including specific steps to be completed. So we will develop tasks where real world phenomena even hazardous accidents can be experienced in a safe learning environment and in the same time we are able to receive valuable information whether these tasks will be effective learning tasks.

\section{ACKNOWLEDGEMENT}

The authors would like to thank everyone who have participated in the development of this training VR technology. This work was supported by Business Finland and Turku University of Applied Sciences.

\section{REFERENCES}

[1] Lauronen, J., Ravyse, W., Salokorpi, M., and Luimula, M. Validation of virtual command bridge training environment -
Comparing the VR-training with ship bridge simulation, In: In Proceedings of the 11th International Conference on Applied Human Factors and Ergonomics (AHFE), San Diego, USA, 16-20 July 2020.

[2] Safety and Shipping review 2019. Allianz global corporate \& specialty. Allianz Corporation. May 2018

[3] Zghyer, Rami \& Ostnes, Runar. (2019). Opportunities and Challenges in Using Ship-Bridge Simulators in Maritime Research., $\begin{array}{llll}\text { August } & 1 \text { 2019. } & \text { DOI }\end{array}$ https://www.researchgate.net/publication/335203192_Opportuniti es_and_Challenges_in_Using_Ship_Bridge_Simulators_in_Mariti me_Research

[4] Virtual reality enables immersive training. January 14, 2020. DOI $=$ https: $/ /$ www.rivieramm.com/news-content-hub/news-contenthub/virtual-reality-enables-immersive-training-57458

[5] Hayhoe, M., \& Ballard, D. (2014). Modeling task control of eye movements. Current Biology, 24(13), R622-R628.

[6] Hayhoe, M., \& Ballard, D. (2005). Eye movements in natural behavior. Trends in cognitive sciences, 9(4), 188-194.

[7] Hämäläinen, H., Izullah, F.R., Koivisto, M., Takio, F., and luimula, M. The Right-side Perceptual Bias in Aging Determined in a Laboratory and during a Virtual Driving Task, Scandinavian Journal of Psychology, Vol. 59, 2018, pp. 32-40.

[8] Luimula, M., Besz, A., Pitkäkangas, P., Suominen, T., Smed, J., Izullah, F.R., and Hämäläinen, H. Virtual Evaluation Tool in Driving Inspection and Training, In: Proceedings of the 5th IEEE Conference on Cognitive Infocommunications, Gyor, Hungary, 2015, pp. 57-60.

[9] Markopoulos, E. and Luimula, M. Immersive Safe Oceans Technology: Developing Virtual Onboard Training Episodes for Maritime Safety. Future Internet 2020, 12, 80.

[10] Diaz, G., Cooper, J., Rothkopf, C., \& Hayhoe, M. (2013). Saccades to future ball location reveal memory-based prediction in a virtualreality interception task. Journal of vision, 13(1), 20-20.

[11] Rayner, K. (2009). The 35th Sir Frederick Bartlett Lecture: Eye movements and attention in reading, scene perception, and visual search. Quarterly journal of experimental psychology, 62(8), 14571506.

[12] Olk, B., Dinu, A., Zielinski, D. J., \& Kopper, R. (2018). Measuring visual search and distraction in immersive virtual reality. Royal Society open science, 5(5), 172331.

[13] Kit, D., Katz, L., Sullivan, B., Snyder, K., Ballard, D., \& Hayhoe, M. (2014). Eye movements, visual search and scene memory, in an immersive virtual environment. PLoS One, 9(4), e94362.

[14] Helbing, J., Draschkow, D., \& Võ, M. L. H. (2020). Search superiority: Goal-directed attentional allocation creates more reliable incidental identity and location memory than explicit encoding in naturalistic virtual environments. Cognition, 196, 104147.

[15] Markopoulos, E., Lauronen, J., Luimula, M., Lehto, P. and Laukkanen, S. Maritime safety education with VR technology (MarSEVR). In Proceedings of the 9th IEEE Conference on Cognitive Infocommunications, Naples, Italy, 23-25 October 2019; pp. 283-288.

[16] Virtual Reality Finnish Disruptive Innovation transforms Maritime Safety Training and Education. 30 October, 2019. DOI = https:/www.allaboutshipping.co.uk/2019/10/30/191293/

[17] Hevner, A.R.; March, S.T.; Ram, J.P. Design science in information systems research. MIS Q. 2004, 28, 75, doi:10.2307/25148625.

[18] Norman, D., and Nielsen, J. The Definition of User Experience. DOI $=$ https://www.nngroup.com/articles/definition-userexperience/

[19] Nielsen Norman Group. Usability 101: Introduction to Usability. DOI $=$ https://www.nngroup.com/articles/usability-101introduction-to-usability/

[20] Varjo. Industrial-Strength Eye Tracking in Varjo's headset (n.d) DOI $=\mathrm{https} / /$ varjo.com/blog/industrial-strength-eye-tracking-invarjo/ 